compared with wild-type cells. Importantly, though MMS is toxic to trm $9-\Delta$ cell growth, this can be largely reversed by overexpressing $R N R$ genes, which illustrates a link between the DNA damage phenotype and the growth limitation associated with reduced RNR protein in the trm $9-\Delta$ cells. More generally, they identified over- and under-represented codons in mRNAs associated with known functional themes, including environmental stress response, protein synthesis and energy metabolism ${ }^{3}$.

Deficiency of any cognate tRNA would slow translational elongation. Based on their data, Begley et al. hypothesized that unmodified tRNA $_{\text {UCU }}^{\text {Arg }}$ and tRNA ${ }_{\underline{U} U C}^{\text {Glu }}$ have decreased cognate decoding activity. Yet AGA is not a rare codon in yeast, and general translation activity was similar in trm9- $\Delta$ and wild-type cells, which suggests no gross decrease in general decoding capacity ${ }^{3}$. trm $9-\Delta$ cells appear not to have a decrease in levels of tRNA ${ }_{\mathrm{UUC}}^{\text {Glu }}$ (ref. 5), which is therefore apparently not degraded, unlike tRNAs undermodified at other positions ${ }^{8}$. It is possible that inappropriate extension of the wobble capacity of the hypomodified tRNAs might shift them to near- or noncognate codons ${ }^{6}$, thereby making them less available for cognate codons.
In either case, $\operatorname{Trm} 9 \mathrm{p}$ would increase the

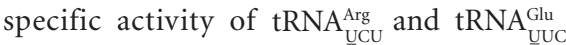
for cognate decoding of $R \bar{N} R 1$ mRNA (and others with cognate codon overabundance), which explains how Trm9p decreases sensitivity to MMS (Fig. 1c) ${ }^{3}$. The potential effects of codon context and a role for the elongation factor Yef $3 p$ in this mode of translational enhancement remain to be determined. Intriguingly, as noted ${ }^{7}$, though various U34 modifications are found in all kingdoms of life on Earth, $\mathrm{mcm}^{5} \mathrm{U}$ and $\mathrm{mcm}^{5} \mathrm{~s}^{2} \mathrm{U}$ appear limited to eukaryotes, which suggests complex functions. Moreover, Trm9-mediated methylation is potentially reversible ${ }^{7}$.

The breakthrough from Begley et al. ${ }^{3}$ is two-fold: that clusters of mRNAs have unusually high abundances of specific codons and that decoding activity of specific tRNAs can be keyed to codon usage in a genetic program. While nature chose to link decoding to anticodon modification and codon usage in this yeast stress response, the possibility that other tRNA-related activities may be involved in other systems should be suspected. Of note are reports that alterations to tRNA processing, modification and subcellular transport can signal or result from stress, including MMS, nutrient depri- vation and Elongator-sensitive zymocin toxicity $^{4,5,9-11}$. Although the chemical state of U34 and the decoding capacity of hypomodified tRNA ${ }_{\bar{U}}^{\mathrm{Arg}}$ and $\mathrm{tRNA}_{\overline{\mathrm{UUC}}}^{\mathrm{Glu}}$ in trm9- $\Delta$ cells remain to be determine $\bar{d}$, the results point to exciting new means of genetic control. It will be interesting to know whether changes in the relative amount and/or decoding capacity of other subsets of tRNAs - as can occur via alterations in tRNA splicing, transport, modification or other processes ${ }^{9-11}$ —are keyed to codon usage.

1. Dittmar, K.A., Goodenbour, J.M. \& Pan, T. PLoS Genet. 2, e221 (2006)

2. Kimchi-Sarfaty, C. et al. Science $\mathbf{3 1 5}, 525-528$ (2007).

3. Begley, U. et al. Mol. Cell 28, 860-870 (2007).

4. Lu, J., Huang, B., Esberg, A., Johansson, M.J. \& Bystrom, A.S. RNA 11, 1648-1654 (2005).

5. Jablonowski, D., Zink, S., Mehlgarten, C., Daum, G. \& Schaffrath, R. Mol. Microbiol. 59, 677-688 (2006).

6. Agris, P.F. Nucleic Acids Res. 32, 223-238 (2004).

7. Kalhor, H.R. \& Clarke, S. Mol. Cell. Biol. 23, 92839292 (2003).

8. Alexandrov, A. et al. Mol. Cell 21, 87-96 (2006).

9. Qiu, H. et al. Mol. Cell. Biol. 20, 2505-2516 (2000).

10. Shaheen, H.H. \& Hopper, A.K. Proc. Natl. Acad. Sci. USA 102, 11290-11295 (2005).

11. Ghavidel, A. et al. Cell 131, 915-926 (2007).

\title{
Looking at membrane lipids from the inside of the cell
}

\section{J Antoinette Killian}

\section{A novel biosensor developed to visualize phosphatidylserine in intact cells suggests a new role for the anionic lipid in specifying intracellular membranes involved in signaling events.}

Eukaryotic cells contain many organelles and hence are full of membranes. Proteins that reside in the cytosol, but that act on membranes, are therefore faced with an important decision: on which of these membranes should they act? The phospholipid phosphatidylserine (PS) could be a decisive factor for targeting of positively charged proteins, because it is the most abundant anionic lipid. Until now it was impossible to directly investigate the role of PS in protein

J. Antoinette Killian is in the Chemical Biology and Organic Chemistry Research Group, Bijvoet Center for Biomolecular Research and Institute of Biomembranes, Department of Chemistry, Faculty of Science, Utrecht University, Padualaan 8, 3584 CH Utrecht,

The Netherlands.

e-mail: J.A.Killian@uu.nl targeting, because there have been no suitable methods to visualize this lipid in intact cells. Yeung et al. ${ }^{1}$ have now developed a fluorescent biosensor that can be expressed in eukaryotic cells and that selectively binds to PS, thereby allowing detection of this lipid on all membranes that are in communication with the cytoplasm. They observed that PS is localized exclusively at the cytoplasmic sides of the plasma membrane and of endocytic compartments, which implies that PS is able to attract specific binding proteins, including signaling proteins, to these membranes. The authors concluded that PS can modulate the localization of signaling proteins in the cell by regulating the membrane surface charge.

The lipid composition of the plasma membrane is asymmetric, with PS being located exclusively in the inner leaflet, exposed to the cytosolic compartment, where it is involved in various signaling processes including activation of protein kinase C (ref. 2). Maintaining this lipid asymmetry requires a delicate balance between the actions of several different proteins involved in lipid transbilayer transport ${ }^{3}$. When cells enter the apoptotic program, PS appears on the outer leaflet of the plasma membrane, and the cells are subsequently recognized and destroyed by macrophages ${ }^{2}$. PS exposure is also a key event in the initiation of the blood clotting cascade $^{2}$.

Given its important physiological roles, it is not surprising that there are many natural proteins that recognize PS. The new PS biosensor developed in this study ${ }^{1}$ is a genetically encoded proteinaceous probe that is expressed in live cells and that consists of a fluorescent reporter molecule linked to lactadherin, a PS-binding protein from milk ${ }^{4}$. 
Lactadherin is a convenient probe because, unlike many other PS-binding proteins, it is able to recognize very low concentrations of PS, it binds PS very specifically, and its action is independent of $\mathrm{Ca}^{2+}$, thus allowing its use under different physiologically relevant conditions.

While PS is expected to be present in many membranes, the authors revealed that PS was accessible to the probe only at the cytoplasmically exposed sides of the plasma membrane and of compartments in the endocytic pathway ${ }^{1}$. This intriguing observation implies that specifically these compartments have docking sites for PSbinding proteins. Because these include important proteins involved in signaling, the findings are consistent with the recent report that plasma membrane receptors may continue signaling even after being taken up by endocytosis for recycling 5 . The authors also compared the localization of PS with that of a series of previously developed and endogeneously expressed surface charge sensors consisting of a cationic sequence with a varying number of charges and a hydrophobic aliphatic chain ${ }^{6}$ (Fig. 1). The results of these colocalization studies suggested that PS is a main determinant for the membrane surface charge. Interestingly, many proteins involved in signaling cascades have properties similar to those of the surface charge sensors. Indeed, the authors observed that such signaling proteins behaved like the sensors and could partition between the different PS-containing membranes depending on their charge. The biological relevance of these observations becomes particularly clear in the experiments that followed, where it was shown that the choice of membrane could be modulated by varying physiological conditions. In particular, reducing the surface charge by changing the transbilayer distribution of PS upon influx of $\mathrm{Ca}^{2+}$ or reducing the positive charge on the protein by phosphorylation of the hydroxyl group of a tyrosine (a common step in signaling cascades) led to relocalization of these proteins to other membranes. These results suggest a new role for PS in signaling by modulation of protein targeting using a simple selection criterion: the amount of surface charge.

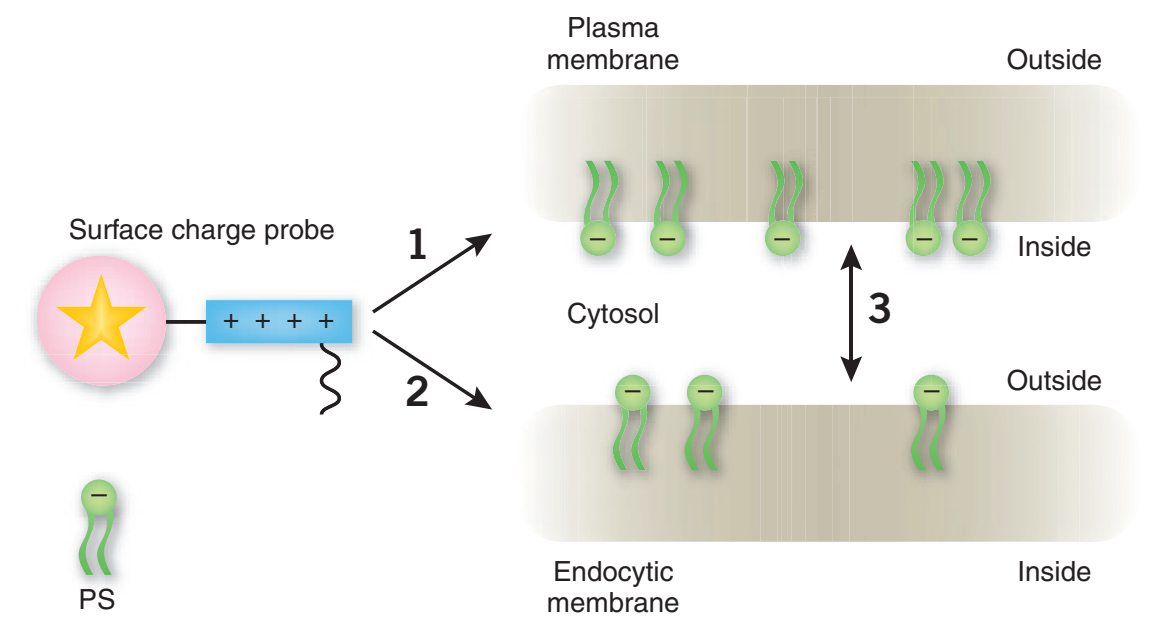

Figure 1 Sensing PS with an endogeneously expressed biosensor. By using a newly developed biosensor for PS, it is shown that this anionic lipid is exposed on the cytoplasmic side of the plasma membrane (top) and (to a lesser extent) on the cytoplasmic side of endocytic compartments within the cell (bottom). Surface charge probes with a fluorescent probe linked to a cationic tail to which a hydrophobic aliphatic chain is attached (left), or proteins involved in signaling that have similar properties, can associate with either monolayer. Partitioning of these proteins between the different membranes is affected by the charge of the protein, with more highly charged proteins favoring the plasma membrane (1) and less charged proteins partitioning significantly to endocytic membranes (2). The partitioning is dynamic (3), and the proteins can be relocalized from the plasma membrane to the endocytotic membrane either by reducing the surface charge of the plasma membrane or by reducing the net positive charge on the protein, for example by phosphorylation.

The development of the new biosensor offers a number of practical applications. For example, it may be useful as a tool to detect changes in PS exposure of membranes in intact cells as a response to environmental changes, to drugs or to genetic changes. It may be applied as diagnostic tool in diseases involving defects in signaling or targeting. It may also be used to discover putative proteins involved in establishing or maintaining lipid asymmetry, to test agents that alter the lipid distribution or to find the molecular basis of diseases related to apoptotic events. A potential drawback in some applications will be that the method is not quantitative. For example, the binding to PS of lactadherin is sensitive to membrane curvature ${ }^{4}$, which may be different for different membranes. Also, the accessibility of the lipids to the protein probe may vary, for example due to shielding by interaction with other proteins, or because of rapid transbilayer movement of the lipids.
The relatively simple method used here will likely not offer possibilities to study localization of other lipids, particularly those in low abundance since binding of an expressed protein could make these lipids unavailable for cellular function. In fact, the relative abundance of PS in membranes may very well be the reason that the probe developed by Yeung et al. ${ }^{1}$ was successful. The results obtained with this probe nicely illustrate how nature can use relatively simple and general concepts, such as electrostatic interactions, to guide key processes like protein targeting.

1. Yeung, T. et al. Science 319, 210-213 (2008).

2. Vance, J.E. \& Steenbergen, R. Prog. Lipid Res. 44 207-234 (2005).

3. Holthuis, J.C.M. \& Levine, T.P. Natl. Rev. 6, 209-220 (2005).

4. Shi, J., Heegaard, C.W., Rasmussen, J.T. \& Gilbert, G.E. Biochim. Biophys. Acta 1667, 82-90 (2004).

5. Miaczynska, M., Pelkmans, L. \& Zerial, M. Curr. Opin. Cell Biol. 16, 400-406 (2004).

6. Roy, M.O., Leventis, R. \& Silvius, J.R. Biochemistry 39, 8298-8307 (2000). 